\title{
A Generation Expansion Planning Model of a Strategic Electricity Generating Firm
}

\author{
M. R. Hesamzadeh, Member, IEEE, and M. Amelin, Member, IEEE
}

\begin{abstract}
This paper derives a mathematical structure for investment decisions of a profit-maximising and strategic producer in liberalised electricity markets. The paper assumes a Cournot producer in an energy market with nodal pricing regime. The Cournot producer is assumed to have revenue from selling energy to the pool. The investment problem of the strategic producer is modelled through a leader-follower game in applied mathematics. The leader is the strategic producer seeking the optimal mix of its investment technologies and the follower is a stochastic estimator. The stochastic estimator forecasts the reactions of other producers in the market in response to the investment decisions of the producer in question. The stochastic estimator takes the investment decisions of the producer and it calculates the stochastic prices. The mathematical structure is a stochastic linear bilevel programming problem. This problem is reformulated as a stochastic MILP problem which can be solved using the commercially available software packages. Finally, the developed mathematical structure is applied to a six-node example system to highlight the strengths of the whole approach.
\end{abstract}

Index Terms-- Generation expansion planning, Mixed-integer linear programming, Uncertainty

\section{INTRODUCTION}

G ENERATION investment has been carefully monitored from the beginning of introducing competition in the generation sector of the electricity industry, [1].

The strategic producers are competing with each other in an oligopoly market to have the highest share and profit from the electricity market. They bid strategically to the market and try to invest their new generation capacities in high-profit regions of the market. To optimally allocate their new generation capacities, the producers need to take into account many scenarios of future loads, generations, transmission capacities, and other generating companies' strategic reactions, [2], [6].

In the liberalized electricity market around the world, a number of policies have sought to directly and indirectly increase the penetration of wind generation and other intermittent generation technologies, [7], [9] . However, highly variable generation technologies, such as wind, increase the uncertainty level in the investment decisions of a producer, [9], [11].

The increasing level of uncertainties in the generation sector raises the following two issues:

(a) How should an electricity generating firm invest in new generating units in this risky environment?

(b) Given the increased level of uncertainties, does the generation investment pattern follow an efficient path?

This paper focuses on the first issue. It derives a stochastic mixed-integer linear programming problem for risky investment of a producer. The mathematical structure finds the high-profit areas of the market for generation investment. It takes into account the new sources of uncertainties such as high variable generation technologies in its assessment process.

Reference [12] presents a game-theoretic model for generation investment problem. It models the generating companies as the Cournot players and it finds the equilibrium of the strategic investment problem under these conditions. An incomplete information game is used in [13] to model the strategic generation investment.

Cournot competition is used in [14] to model the behaviour of competing electricity generating firms.

Reference [15] proposes a payoff-based approach for generation expansion planning to meet the economic and efficiency requirements of the electricity market.

Reference [16] presents three models for generation expansion planning. The first proposes a perfect competitive equilibrium, the second is the open loop Cournot equilibrium and the third one is the closed loop cournot equilibrium.

Reference [17] reviews the literature on generation investment problem.

This paper develops and efficiently solves a stochastic mathematical structure for generation expansion planning of an electricity generating firm. The rest of this paper is organised as follows: section II explains the mathematical formulation. The solution method is discussed in section III. Section IV tests the developed mathematical structure on a six-node example system. Section V concludes the paper.

\section{THE MATHEMATICAL FORMULATION}

The optimal generation investment problem of an electricity generating firm is modelled using the static version of the leader-follower game in applied mathematics. The leader is the electricity generating firm and the follower is a player termed "Stochastic Estimator". The stochastic estimator finds the reaction of the electricity market players to the investment decisions of the electricity generating firm. 


\section{A. Electricity Generating Firm}

Suppose $A$ is the set of all generating units available for the electricity generating firm, $k$, and $B$ is the future generating units ( $B \subset A$ ). The investment decision problem of electricity generating firm $k$ is formulated in (1).

$$
\begin{aligned}
& \operatorname{Max}_{\tilde{q}_{j}, \tilde{z}_{j}} \Pi_{t}=E\left[\sum_{j=1}^{N_{A}}\left(\tilde{p}_{j}-c_{j}\right) \tilde{q}_{j}+\sum_{j=1}^{N_{B}} b_{j} \tilde{z}_{j}\right] \\
& \text { s.t. } \\
& 0 \leq \tilde{q}_{j} \leq Q_{j} \quad j=1, \ldots, N_{A} \\
& 0 \leq \tilde{q}_{j} \leq \tilde{z}_{j} Q_{j} \quad j=1, \ldots, N_{B} \\
& f\left(\tilde{z}_{j}=k\right)=x_{j}^{k}\left(1-x_{j}\right)^{1-k} \quad k \in\{0,1\}
\end{aligned}
$$

In (1), $\tilde{p}_{j}$ is the price at the connection point of generating unit $j$ in $\$ / M W, \tilde{q}_{j}$ is the dispatched quantity in $M W, c_{j}$ is the marginal cost of production in $\$ / M W, b_{j}$ is the investment cot of generating unit $j, \quad \tilde{z}_{j}$ is the binary decision variable with Bernoulli distribution, $\tilde{q}_{j}$ is the offer quantity of generating unit $\mathrm{j}$ in $M W$, and $\tilde{Q}_{j}$ is the maximum generation capacity of generating unit $\mathrm{j}$ in $M W . E[*]$ is the expectation operator and variables with $\sim$ sign are random variables.

\section{B. Stochastic Estimator}

The stochastic estimator finds the reaction of the electricity market to each investment decision of the electricity generating firm. In doing this, it uses the available market data to estimate the reactions of the other generators in the market.

Fig. 1 shows the block diagram of the stochastic estimator.

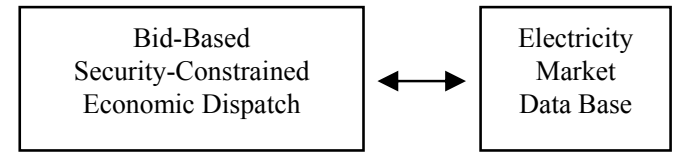

Fig. 1 The block diagram of the stochastic estimator function

The bid-based security-constrained economic dispatch is formulated in (2).

$$
\begin{aligned}
& \operatorname{Max}_{q_{j}}-\sum_{j \in G} \tilde{c}_{j} \tilde{q}_{j} \\
& \text { s.t. } \\
& \qquad \sum_{j=1}^{N-1} B_{k j} \tilde{\theta}_{j}=\sum_{j \in G_{k}} \tilde{q}_{j}-\sum_{j \in D_{k}} \tilde{d}_{j} \quad k=1, \ldots, N \\
& \quad-F_{l} \leq \sum_{j=1}^{N-1} H_{l j} \tilde{\theta}_{j} \leq F_{l} \quad l=1, \ldots, L \\
& \quad 0 \leq \tilde{q}_{j} \leq Q_{j} \quad j=1, \ldots, N_{G}
\end{aligned}
$$

The interaction of the electricity generating firm with the stochastic estimator is depicted in Fig. 2.

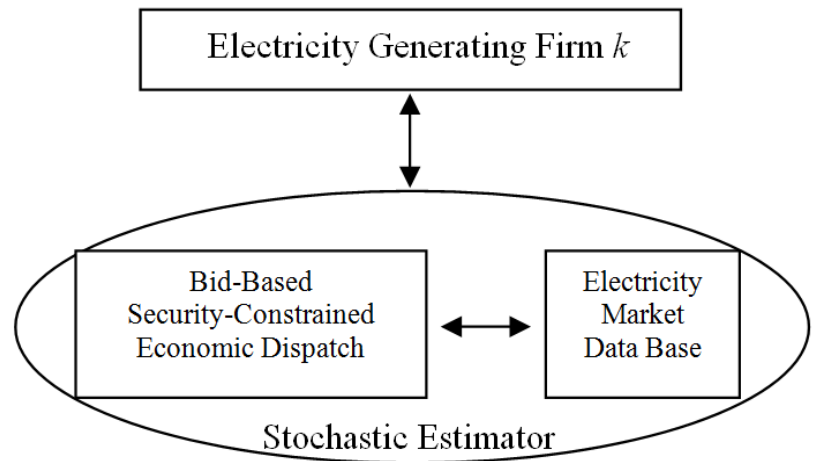

Fig. 2 The interaction of the electricity generating firm with the stochastic estimator

In (3), the interaction of the electricity generating firm and the stochastic estimator is mathematically modelled.

$$
\begin{aligned}
& \operatorname{Max}_{\tilde{q}_{j}, \tilde{z}_{j}} \Pi_{t}=E\left[\sum_{j=1}^{N_{A}}\left(\tilde{p}_{j}-c_{j}\right) \tilde{q}_{j}+\sum_{j=1}^{N_{B}} b_{j} \tilde{z}_{j}\right] \\
& \text { s.t. } \\
& 0 \leq \underline{\tilde{q}}_{j} \leq Q_{j} \quad j=1, \ldots, N_{A} \\
& 0 \leq \tilde{q}_{j} \leq \tilde{z}_{j} Q_{j} \quad j=1, \ldots, N_{B} \\
& f\left(\tilde{z}_{j}=k\right)=x_{j}^{k}\left(1-x_{j}\right)^{1-k} \quad k \in\{0,1\} \\
& \operatorname{Max}_{q_{j}}-\sum_{j \in G} \tilde{c}_{j} \tilde{q}_{j} \\
& \text { s.t. } \\
& \begin{array}{l}
\sum_{j=1}^{N-1} B_{k j} \tilde{\theta}_{j}=\sum_{j \in G_{k}} \tilde{q}_{j}-\sum_{j \in D_{k}} \tilde{d}_{j} \quad k=1, \ldots, N \\
-F_{l} \leq \sum_{j=1}^{N-1} H_{l j} \tilde{\theta}_{j} \leq F_{l} \quad l=1, \ldots, L \\
0 \leq \tilde{q}_{j} \leq \tilde{q}_{j} \quad j=1, \ldots, N_{G}
\end{array}
\end{aligned}
$$

The mathematical structure in (3) is a stochastic two-level, mixed-integer linear programming problem.

\section{THE MATHEMATICAL SOLUTION}

To model the endogenous prices in the leader's investment problem, the mathematical formulation of the stochastic estimator is replaced by its Karush-Kuhn-Tucker optimality conditions and then further transformed into mixed integer programming problem. This is done by exploiting the disjunctive nature of the complementary slackness conditions. The nonlinear terms in the producer's objective function are removed employing the strong duality theorem in convex optimisation.

Subsections C, D, and E explain these three steps.

\section{The Karush-Kuhn-Tucker optimality conditions of the stochastic estimator formulation}

The Lagrangian of the optimisation problem in (2) is derived in (4). 


$$
\begin{aligned}
& L=-\sum_{j \in G} \tilde{c}_{j} \tilde{q}_{j}+ \\
& \sum_{k=1}^{N} p_{k}\left(-\sum_{j=1}^{N-1} B_{k j} \tilde{\theta}_{j}+\sum_{j \in G_{k}} \tilde{q}_{j}-\sum_{j \in D_{k}} \tilde{d}_{j}\right)+ \\
& \sum_{l=1}^{L} u_{l}^{u p}\left(F_{l}-\sum_{j=1}^{N-1} H_{l j} \tilde{\theta}_{j}\right)+\sum_{l=1}^{L} u_{l}^{\text {lower }}\left(F_{l}+\sum_{j=1}^{N-1} H_{l j} \tilde{\theta}_{j}\right)+ \\
& \sum_{j=1}^{N_{G}} v_{l}^{u p}\left(\underline{q}_{j}-\tilde{q}_{j}\right)+\sum_{j=1}^{N_{G}} v_{l}^{\text {lower }} \tilde{q}_{j}
\end{aligned}
$$

Using the Lagrangian (L) above, the Karush-Kuhn-Tucker optimality conditions can be derived as in (5).

$$
\begin{aligned}
& \sum_{j=1}^{N-1} B_{k j} \tilde{\theta}_{j}=\sum_{j \in G_{k}} \tilde{q}_{j}-\sum_{j \in D_{k}} \tilde{d}_{j} \quad k=1, \ldots, N \\
& -F_{l} \leq \sum_{j=1}^{N-1} H_{l j} \tilde{\theta}_{j} \leq F_{l} \quad l=1, \ldots, L \\
& 0 \leq \tilde{q}_{j} \leq \tilde{q}_{j} \quad j=1, \ldots, N_{G} \\
& -\tilde{c}_{j}+\sum_{k=1}^{N} p_{k} r_{\left(k, N_{G}\right)}-v_{j}^{u p}+v_{j}^{\text {low }}=0 \quad j=1, \ldots, N_{G} \\
& \sum_{k=1}^{N} p_{k} B_{(k, m)}-\sum_{l=1}^{L} u_{l}^{u p} H_{(l, m)}+ \\
& \sum_{l=1}^{L} u_{l}^{\text {low }} H_{(l, m)}=0 \quad m=1, \ldots, N-1 \\
& u_{l}^{\text {up }}\left(F_{l}-\sum_{j=1}^{N-1} H_{l j} \tilde{\theta}_{j}\right)=0 \quad l=1, \ldots, L \\
& u_{l}^{\text {lower }}\left(F_{l}+\sum_{j=1}^{N-1} H_{l j} \tilde{\theta}_{j}\right)=0 \quad l=1, \ldots, L \\
& v_{j}^{\text {up }}\left(\tilde{q}_{j}-\tilde{q}_{j}\right)=0 \quad j=1, \ldots, N_{G} \\
& v_{j}^{\text {lower }} \tilde{q}_{j}=0 \quad j=1, \ldots, N_{G}
\end{aligned}
$$

\section{The disjunctive nature of the complementary slackness conditions}

The only nonlinear terms in the Karush-Kuhn-Tucker optimality conditions (5) are the complementary slackness conditions. These are linearized in (6) using their disjunctive nature.

$$
\begin{aligned}
& -A\left(1-y_{l}^{u p}\right) \leq\left(F_{l}-\sum_{j=1}^{N-1} H_{l j} \tilde{\theta}_{j}\right) \leq \AA\left(1-y_{l}^{u p}\right) \\
& l=1, \ldots, L \\
& -A y_{l}^{u p} \leq u_{l}^{u p} \leq A y_{l}^{u p} \quad l=1, \ldots, L \\
& -A\left(1-y_{l}^{l o w}\right) \leq\left(F_{l}+\sum_{j=1}^{N-1} H_{l j} \tilde{\theta}_{j}\right) \leq A\left(1-y_{l}^{l o w}\right) \\
& \quad l=1, \ldots, L
\end{aligned}
$$

$$
\begin{aligned}
& -A y_{l}^{\text {low }} \leq u_{l}^{\text {low }} \leq A y_{l}^{\text {low }} \quad l=1, \ldots, L \\
& -A\left(1-w_{l}^{u p}\right) \leq\left(\underline{\tilde{q}}_{j}-\tilde{q}_{j}\right) \leq \AA\left(1-w_{l}^{u p}\right) \quad j=1, \ldots, N_{G} \\
& -\AA w_{l}^{\text {up }} \leq v_{j}^{u p} \leq \AA w_{l}^{\text {up }} \quad j=1, \ldots, N_{G} \\
& -A\left(1-w_{l}^{\text {low }}\right) \leq \tilde{q}_{j} \leq \AA\left(1-w_{l}^{\text {low }}\right) \quad j=1, \ldots, N_{G} \\
& -A w_{l}^{\text {low }} \leq v_{j}^{\text {lower }} \leq \AA w_{l}^{\text {low }} \quad j=1, \ldots, N_{G}
\end{aligned}
$$

Where in (6), $A$ is a big number and $y$, and $w$ are binary variables.

\section{E. The Strong Duality Theorem in Convex Optimisation Theory}

Substituting (6) in (5) yields to a set of linear constraints for optimisation problem (3). The only nonlinear term is the term $\left(\tilde{p}_{j}-c_{j}\right) \tilde{q}_{j}$ in the objective function. Since the inner optimisation problem in (3) is a linear programming problem, the strong duality theorem holds.

$$
\begin{aligned}
& -\tilde{c}_{j}+\sum_{k=1}^{N} p_{k} r_{(k, j)}-v_{j}^{u p}+v_{j}^{\text {low }}=0 \stackrel{\times q_{j}(j \in A)}{\longrightarrow} \\
& -\sum_{j=1}^{N_{G}} \tilde{c}_{j} \sum_{j \in A} r_{(k, j)} q_{j}+\sum_{k=1}^{N} p_{k} \sum_{j \in A} r_{(k, j)} q_{j} \\
& -\sum_{j=1}^{N_{G}} v_{j}^{u p} \sum_{j \in A} r_{(k, j)} \underline{\tilde{q}}_{j}=0 \rightarrow \\
& \Pi_{t}=\sum_{j=1}^{N_{G}} v_{j}^{u p} \sum_{j \in A} r_{(k, j)} \underline{q}_{j}
\end{aligned}
$$

Strong duality condition:

$$
\begin{aligned}
& \sum_{i=1}^{N} p_{i} \sum_{j=1}^{N_{D}} s_{i, j} d_{j}+\sum_{i=1}^{N_{L}}\left(u_{i}^{u p}+u_{i}^{\text {low }}\right) F_{i}+
\end{aligned}
$$

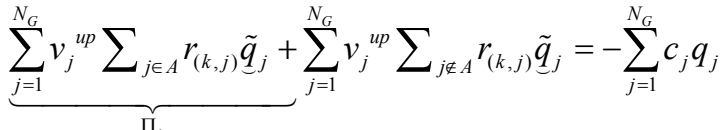

Equation (8) yields to the linear formulation in (9) for the profit function of electricity generating firm $k$.

$$
\begin{aligned}
& \sum_{i=1}^{N} p_{i} \sum_{j=1}^{N_{D}} s_{i, j} d_{j}+\sum_{i=1}^{N_{L}}\left(u_{i}^{u p}+u_{i}^{\text {low }}\right) F_{i}+ \\
& \sum_{j=1}^{N_{G}} v_{j}^{u p} \sum_{j \notin A} r_{(k, j)} \underline{q}_{j}+\sum_{j=1}^{N_{G}} c_{j} q_{j}=-\Pi_{t}
\end{aligned}
$$

By substituting (5), (6), and (9) in (3), the final mathematical structure is derived. This structure is presented in (10). 


$$
\operatorname{Max}_{\tilde{q}_{j}, \tilde{z}_{j}} \Pi_{t}=E\left[\begin{array}{c}
-\sum_{i=1}^{N} p_{i} \sum_{j=1}^{N_{D}} s_{i, j} d_{j}-\sum_{i=1}^{N_{L}}\left(u_{i}^{u p}+u_{i}^{\text {low }}\right) F_{i}- \\
\sum_{j=1}^{N_{G}} v_{j}^{u p} \sum_{j \notin A} r_{(k, j)} \tilde{q}_{j}-\sum_{j=1}^{N_{G}} c_{j} q_{j}+\sum_{j=1}^{N_{B}} b_{j} \tilde{z}_{j}
\end{array}\right]
$$

s.t.

$$
\begin{aligned}
& 0 \leq \underline{q}_{j} \leq Q_{j} \quad j=1, \ldots, N_{A} \\
& 0 \leq \tilde{q}_{j} \leq \tilde{z}_{j} Q_{j} \quad j=1, \ldots, N_{B} \\
& f\left(\tilde{z}_{j}=k\right)=x_{j}^{k}\left(1-x_{j}\right)^{1-k} \quad k \in\{0,1\} \\
& \sum_{j=1}^{N-1} B_{k j} \tilde{\theta}_{j}=\sum_{j \in G_{k}} \tilde{q}_{j}-\sum_{j \in D_{k}} \tilde{d}_{j} \quad k=1, \ldots, N \\
& -F_{l} \leq \sum_{j=1}^{N-1} H_{l j} \tilde{\theta}_{j} \leq F_{l} \quad l=1, \ldots, L \\
& 0 \leq \tilde{q}_{j} \leq \tilde{q}_{j} \quad j=1, \ldots, N_{G} \\
& -\tilde{c}_{j}+\sum_{k=1}^{N} p_{k} r_{\left(k, N_{G}\right)}-v_{j}^{u p}+v_{j}^{\text {low }}=0 \quad j=1, \ldots, N_{G} \\
& \sum_{k=1}^{N} p_{k} B_{(k, m)}-\sum_{l=1}^{L} u_{l}^{u p} H_{(l, m)}+ \\
& \sum_{l=1}^{L} u_{l}^{l o w} H_{(l, m)}=0 \quad m=1, \ldots, N-1 \\
& -\AA\left(1-y_{l}^{u p}\right) \leq\left(F_{l}-\sum_{j=1}^{N-1} H_{l j} \tilde{\theta}_{j}\right) \leq \AA\left(1-y_{l}^{u p}\right) \\
& l=1, \ldots, L \\
& -A y_{l}^{u p} \leq u_{l}^{u p} \leq A y_{l}^{u p} \quad l=1, \ldots, L \\
& -\AA\left(1-y_{l}^{\text {low }}\right) \leq\left(F_{l}+\sum_{j=1}^{N-1} H_{l j} \tilde{\theta}_{j}\right) \leq \AA\left(1-y_{l}^{\text {low }}\right) \\
& l=1, \ldots, L \\
& -A y_{l}^{\text {low }} \leq u_{l}^{\text {low }} \leq A y_{l}^{\text {low }} \quad l=1, \ldots, L \\
& -\AA\left(1-w_{l}^{u p}\right) \leq\left(\tilde{q}_{j}-\tilde{q}_{j}\right) \leq \AA\left(1-w_{l}^{u p}\right) \quad j=1, \ldots, N_{G} \\
& -A w_{l}^{u p} \leq v_{j}^{u p} \leq A w_{l}^{u p} \quad j=1, \ldots, N_{G} \\
& -\AA\left(1-w_{l}^{\text {low }}\right) \leq \tilde{q}_{j} \leq \AA\left(1-w_{l}^{\text {low }}\right) \quad j=1, \ldots, N_{G} \\
& -A w_{l}^{\text {low }} \leq v_{j}^{\text {lower }} \leq A w_{l}^{\text {low }} \quad j=1, \ldots, N_{G}
\end{aligned}
$$

\begin{tabular}{|c|c|c|c|c|}
\hline GenCo & Generator & $\begin{array}{c}\mathrm{g}_{\max } \\
(\mathrm{MW})\end{array}$ & $\begin{array}{c}\text { Deviation } \\
(\mathrm{MW})\end{array}$ & c $(\$ / M W)$ \\
\hline $\mathrm{A}$ & 1(Existing) & 220 & 200 & 12 \\
\hline B & 2 (Future) & 180 & - & 14 \\
\hline $\mathrm{B}$ & 3 (Existing) & 460 & - & 20 \\
\hline \multirow[t]{2}{*}{$\mathrm{C}$} & 4 (Future) & 600 & - & 35 \\
\hline & Total & 1460 & & \\
\hline
\end{tabular}

Optimisation problem in (10) is a stochastic mixed-integer linear programming problem.

In this paper, we use the Monte Carlo technique to handle the stochastic variables in (10).

\section{THE SIX-NODE EXAMPLE SYSTEM}

To evaluate the stochastic MILP formulation derived in (10), a six-node example system is carefully modified and studied. The key parameters of the system are presented in Tables I through III. For this study the investment cost of generator 2 is assumed to be $\$ 200$.
TABLE I GENERATORS’ DATA

\begin{tabular}{c|c|c}
\multicolumn{3}{|c}{ TABLE II RETAILERS' DATA } \\
\hline Retailer & $\mathrm{d}_{\max }(\mathrm{MW})$ & Deviation (MW) \\
\hline $\mathrm{R} 1$ & 80 & 8 \\
\hline $\mathrm{R} 2$ & 130 & 20 \\
\hline $\mathrm{R} 3$ & 40 & 4 \\
\hline $\mathrm{R} 4$ & 160 & 30 \\
\hline R5 & 115 & 25 \\
\hline Total & 525 & \\
\hline
\end{tabular}

\begin{tabular}{c|c|c|c|c}
\multicolumn{5}{c}{ TABLE III TRANSMISSION NETWORK DATA } \\
\hline Line\# & From & To & Reactance (Ohm) & $\begin{array}{c}\text { Limit } \\
\text { (MW) }\end{array}$ \\
\hline 1 & Bus1 & Bus2 & 0.004 & 40 \\
\hline 2 & Bus1 & Bus4 & 0.006 & 50 \\
\hline 3 & Bus1 & Bus5 & 0.002 & 60 \\
\hline 4 & Bus2 & Bus3 & 0.002 & 180 \\
\hline 5 & Bus2 & Bus4 & 0.004 & 50 \\
\hline 6 & Bus2 & Bus6 & 0.003 & 40 \\
\hline 7 & Bus3 & Bus5 & 0.002 & 160 \\
\hline 8 & Bus4 & Bus6 & 0.003 & 100 \\
\hline
\end{tabular}

The single line diagram of the example system is shown in Fig. 3.

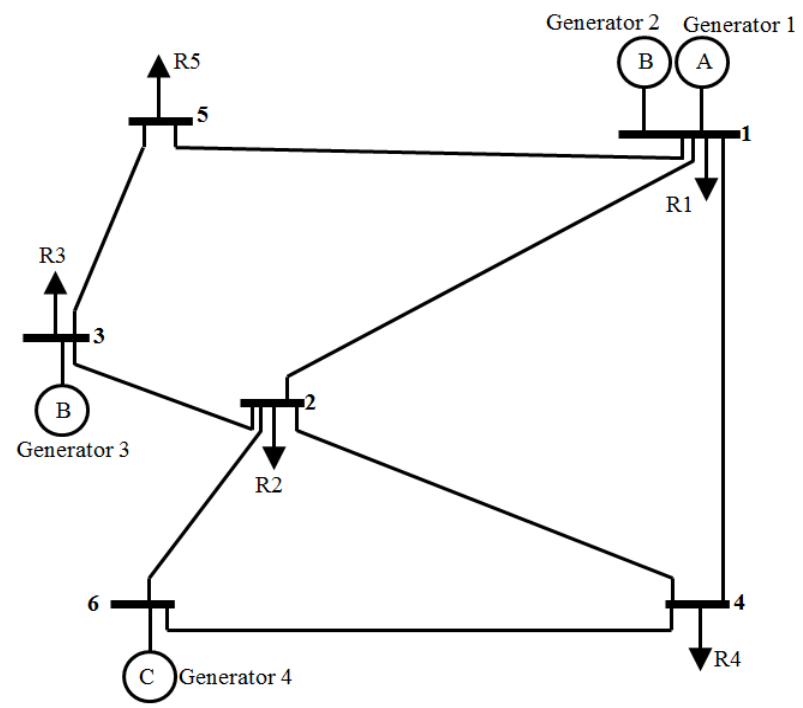

Fig. 3 The six-node example system

There are three generating companies, $\mathrm{A}, \mathrm{B}$, and $\mathrm{C}$ in this example system. GenCo A is the owner of generating unit1. GenCo B owns generating unit 2 as the possible future expansion and generating unit 3 as the existing one. GenCo $\mathrm{C}$ owns generating unit 4 . We use the developed mathematical structure in (10) to evaluate the investment problem of GenCo B. We do this under the following two scenarios; 
(a) Generating unit 1 is modelled as a conventional generator with a fixed generation capacity,

(b) Generating unit 1 is modelled as a wind power generator with varying generation capacity.

Loads in the six-node example system are modelled as random variables with normal distributions. Transmission system is assumed to be very reliable without any contingency.

The mathematical structure in (10) is coded in GAMS software and then it is run for these two scenarios. Table IV shows the mean of nodal prices for the above two scenarios.

The cumulative distribution function of the prices at nodes 1 to 6 in two scenarios of (a) and (b) are illustrated in Fig. 4.

\begin{tabular}{c|c|c}
\multicolumn{3}{c}{ TABLE IV MEAN OF NODAL PRICES IN \$/MW } \\
\hline \multirow{2}{*}{ Node No. } & \multicolumn{2}{|c}{ Mean of nodal prices in \$/MW } \\
\cline { 2 - 3 } & $\begin{array}{c}\text { Without wind } \\
\text { power (a) }\end{array}$ & $\begin{array}{c}\text { With wind power } \\
\text { (b) }\end{array}$ \\
\hline 1 & 104 & 178 \\
\hline 2 & 416 & 423 \\
\hline 3 & 430 & 441 \\
\hline 4 & 228 & 245 \\
\hline 5 & 445 & 460 \\
\hline 6 & 31 & 27 \\
\hline Average & $\mathbf{2 7 5}$ & $\mathbf{2 9 5}$ \\
\hline
\end{tabular}

As in Table IV, the mean of nodal prices at different nodes in scenario (b) is increased as compared to scenario (a). This is mainly because of the increased level of uncertainties in the generation sector. In scenario (b), there are some times that the wind power generator (generating unit 1) is able to produce only very small amounts, around 20MW. At these cases, other more expensive generating units must be dispatched. This results in higher nodal prices and consequently the higher means for nodal prices.

Fig. 4 plots the cumulative distribution functions of the nodal prices at different nodes under two scenarios (a), and (b). The solid lines represent scenario (b) and the dashed lines represent scenario (a). This figure shows the higher prices in wind power scenario as compared to no wind power scenario.

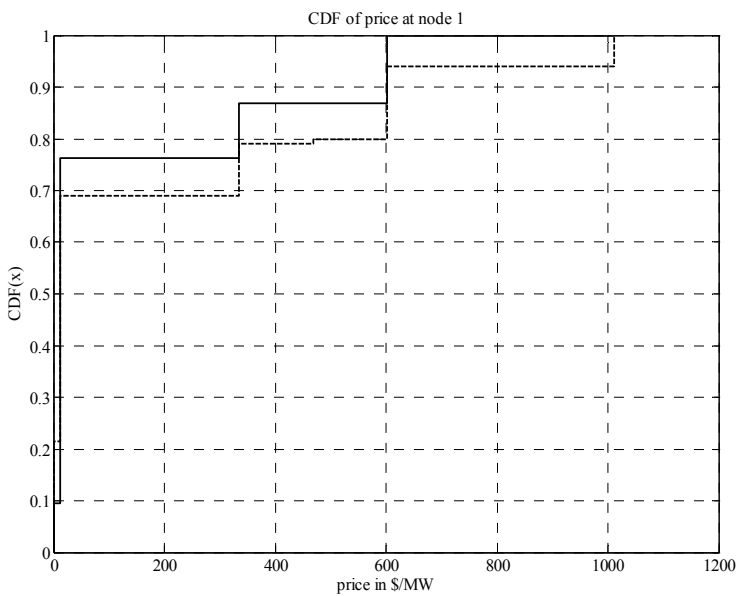

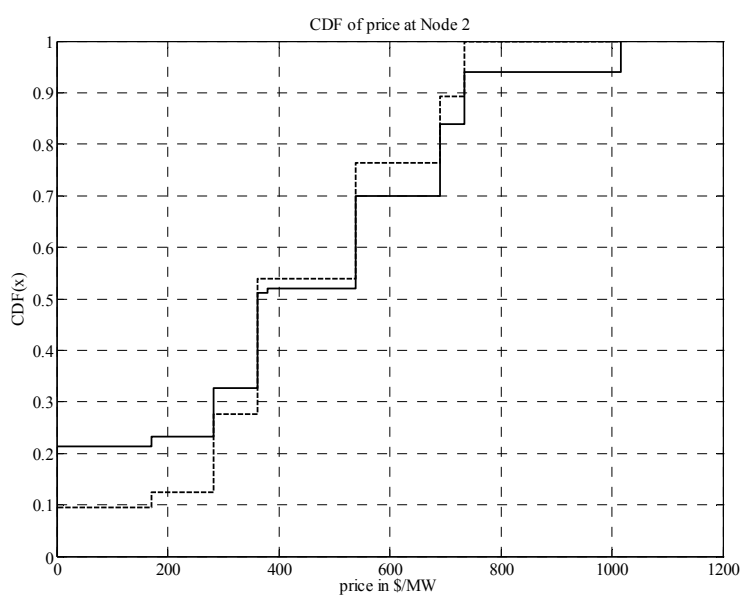
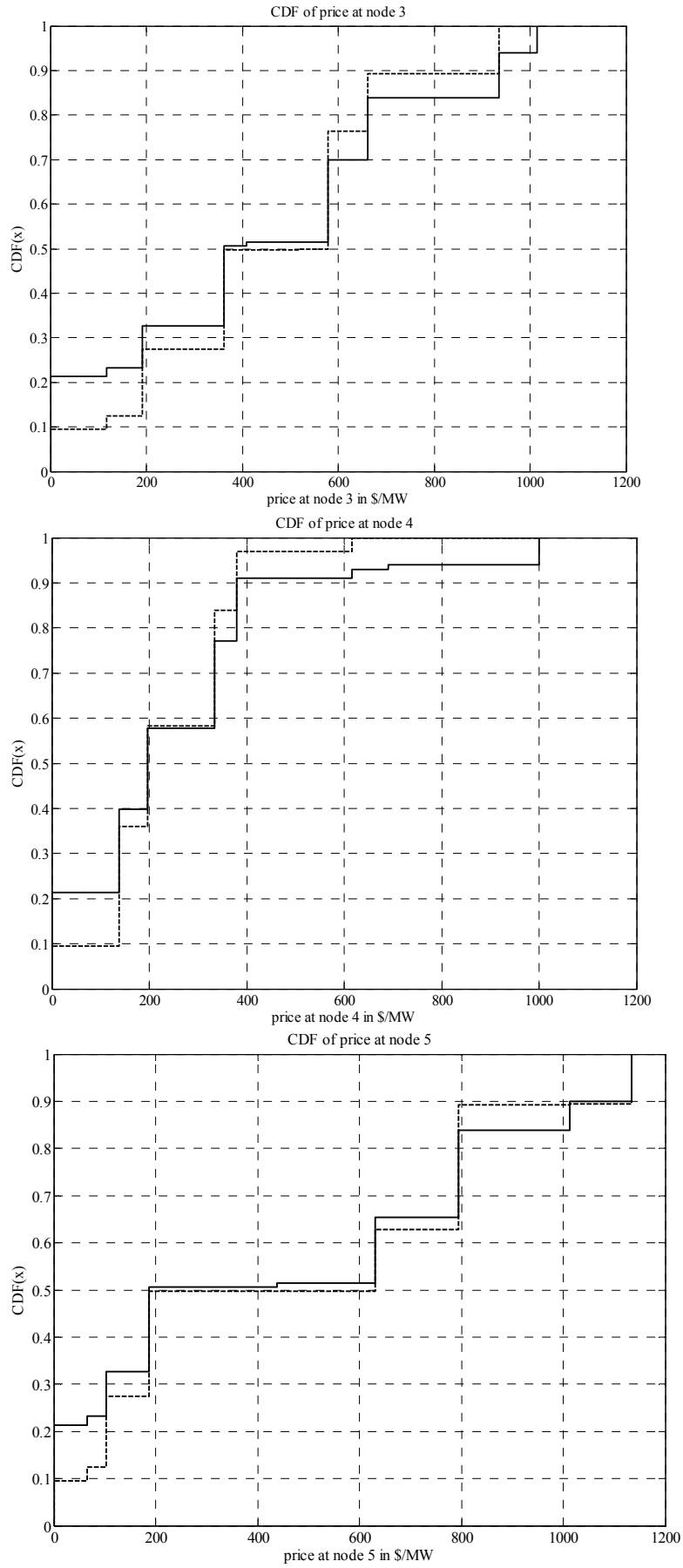


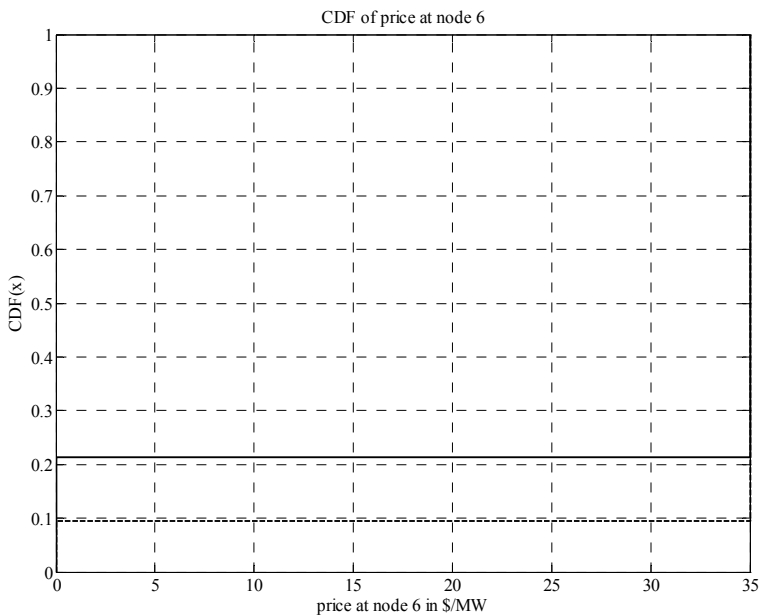

Fig.4 The cumulative distribution function of prices at nodes 1 to 6 (solid lines present scenario (b) and dashed lines present scenario (a))

The cumulative distribution function of the GenCo B profit under two scenarios of (a) and (b) is illustrated in Fig. 5. The mean of GenCo B profit under scenario (a) is $\$ 109,720$ and under scenario (b) is $\$ 140,380$. This shows an increase of $28 \%$ in GenCo B profit.

To analysis this increase in profit, the Bernoulli distribution function of the investment variable of GenCo $\mathrm{B}$, investment variable of generator 2, is calculated in (11).

$$
\begin{aligned}
& f_{a}\left(\tilde{z}_{1}=x\right)=\left\{\begin{array}{cc}
0.092 & x=1 \\
0 & x=0
\end{array}\right. \\
& f_{b}\left(\tilde{z}_{1}=x\right)=\left\{\begin{array}{cc}
0.16 & x=1 \\
0 & x=0
\end{array}\right.
\end{aligned}
$$

From the Bernoulli distribution functions in (11), the probability of investment in generating unit 2 is increased from 0.092 to 0.16 . This means that GenCo B has more incentive to build generating unit 2 in scenario (b) as compared to scenario (a). This is mainly because in scenario (b) there are some times that the wind power generator does not produce much. GenCo B can use these opportunities to run its new generating unit and earn some more profit. This partly explains the $28 \%$ increase in the GenCo B profit.

The analysis above shows the importance of modelling uncertainties in the investment decision of an electricity generating firm. As we saw in this example, the increased level of uncertainties in scenario (b) results in different probabilities of investing in generating unit 2 .

The mathematical structure derived in this paper along with its solution algorithm show promises in assessment of risky investment decisions of an electricity generating firm.

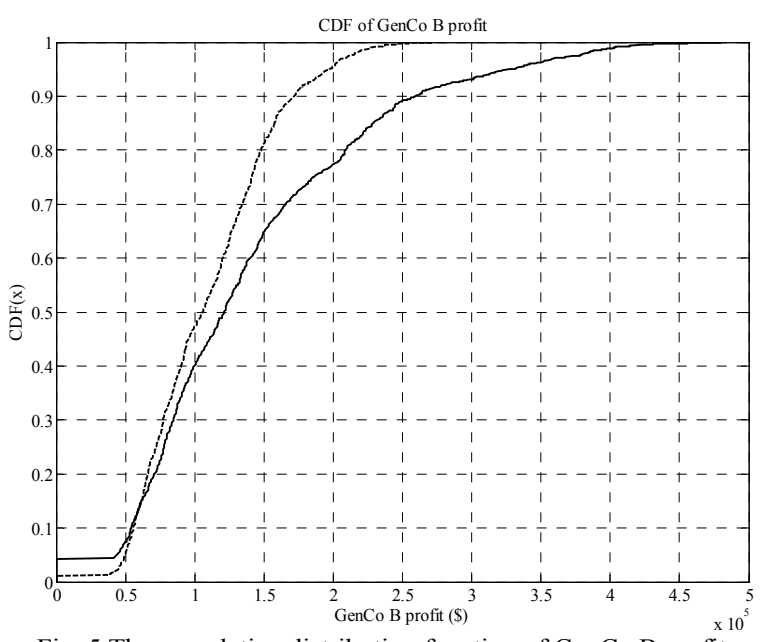

Fig. 5 The cumulative distribution function of GenCo B profit under scenarios b (solid line) and a (dashed line)

\section{CONCLUDING REMARKS}

This paper discusses a mathematical structure and its solution algorithm for risky investment of an electricity generating firm. The derived mathematical structure is a stochastic mixed-integer linear programming, stochastic MILP, problem. It can be efficiently solved using the commercially available optimisation softwares. The derived mathematical structure can be used by a strategic producer to find its optimal mix of generation technologies for investment in the future energy market.

Although the main focus of this paper is on the derivation of the mathematical model, an economic study is carried out using the developed structure and a six-node example system. The results of this paper show that with increased level of uncertainties in the generation sector, the investment decisions of the electricity generating firms change.

The developed mathematical structure shows promises for assessment of expansion planning decisions of an electricity generating firm in this risky environment.

\section{REFERENCES}

[1] A. Bar-Ilan, and W.C. Strange, "Investment lags", American Economic Review, 83 (3), 610-22

[2] P. D. Klemperer, M. A. Meyer, "Supply function equilibria", Econometrica, 1989;57:1243-77.

[3] A. Rudkevich , M. Duckworth, R. Rosen, "Modeling electricity pricing in a deregulated generation industry: the potential for oligopoly pricing in a Poolco", Energy J 1998;19(3):19-48.

[4] S. Borenstein, J. Bushnell, E. Kahn, S. Stoft, "Market power in California electricity markets", Utilities Policy 1995;5(3/4):219-36.

[5] R. Green, "Increasing competition in the british electricity spot market", J Ind Econ 1996;44(2):205-16.

[6] R. Baldick, R. Grant, E. Kahn,"Linear supply function equilibrium: generalizations, application, and limitations", University of California Energy Institute, POWER, PWP-078; 2000.

[7] T. Ackermann, G. Andersson, and L. Söder, "Overview of government and market driven programs for the promotion of renewable power generation", Renewable Energy, 22(2001) pp. 197-204. Elsevier Science Ltd.

[8] L. Söder, "On limits for wind power generation", Published in International Journal of Global Energy Issues, Vol. 21, No. 3, 2004, pp 243254. 
[9] L. Söder, "The value of wind power", Wind Power in Power Systems, January 2005 by Wiley \& Sons, Chichester, UK.

[10] L. Söder, "Modelling approach impact on estimation of integration cost of wind power", Proceedings of 7th IAEE European Energy Conference European Energy Markets in Transition, The Norwegian School of Economics and Business Administration NHH, Bergen, Norway, August 2830th, 2005

[11] H. Outhred, "National Wind Power Study: An estimate of readily accepted wind energy in the National Electricity Market", November 2003

[12] A.S. Chuang, F. Wu, and P. Varaiya, "A Game-Theoretic Model for Generation Expansion Planning: Problem Formulation and Numerical Comparison", IEEE Trans. On Power Systems, Vol. 16, No. 4, November 2001, pp. 885-891

[13] J. Wang, M. Shahidehpour, Z. Li, A. Botterud, "Strategic Generation Capacity Expansion Planning With Incomplete Information", IEEE Trans. On Power Systems, Vol. 24, No. 2, May 2009, pp. 1002-1010

[14] W. J. Yuan, and Y. Smeers, “”, Operations Resaerch, Vol. 47, no. 1, January 1999, pp. 102-112

[15]Y. Fu, and M. Shahidehpour, "Profit-based generation", IMA J. Manage. Math, Vol. 15, no. 4, 2004, pp. 273-289.

[16] F. H. Murphy, Y. Smeer, "Capacity expansion in imperfectly competitive restructured electricity markets", Harvard Electricity Policy Group, Centre for Business and Government, May 2001

[17] A. G. Kagiannas, D. T. Askounis, J. Psarras, "Power generation planning: a survey from monopoly to competition", Electrical Power and Energy Systems 26 (2004), 413-421

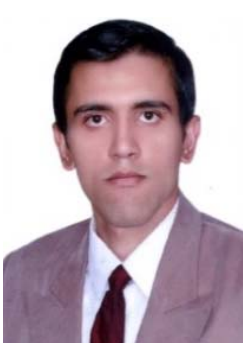

Mohammad R. Hesamzadeh (IEEE-M'10) is a PostDoctoral Fellow at Royal Institute of Technology, Stockholm, Sweden. His Special fields of interests include Economics of Wholesale Electricity Markets, Mathematical Modelling and Computing, and High Performance Computing. Hesamzadeh has a Ph.D. in Electrical Engineering from Swinburne University of Technology, Melbourne, Australia. He is a member of IEEE, a member of IEEE PES System Economics SubCommittee, and a Professional Engineer in Australia.

Mikael Amelin (IEEE-M'07) is an adjunct assistant professor in the Electric Power Systems Lab at the School of Electrical Engineering of the Royal Institute of Technology (KTH) in Stockholm. He received his masters, licentiate and doctors degrees from the same institute in 1997, 2000 and 2004 respectively. His research interests include Monte Carlo techniques, analysis and modelling of electricity markets, as well as rural electrification in developing countries. 